\title{
Treatment of Chronic Lymphocytic Leukemia in the Elderly
}

\author{
*Nahla A. M. Hamed \\ Professor of Hematology, Alexandria University, Egypt \\ Submission: March 10, 2017; Published: March 16, 2017 \\ "Correspondence Address: Nahla A. M. Hamed, Professor of Hematology, Faculty of medicine, Alexandria University, Egypt
}

\begin{abstract}
Elderly patients have a more limited bone marrow reserve in comparison with younger patients and may recover more slowly from drug-related cytopenias. Age-related decline in renal and hepatic function may delay clearance of antileukemic agents cleared by liver and kidney, necessitating their dose reduction. Comorbidities have been postulated to contribute to CLL-unrelated death, facilitate toxicity to CLL treatment, predispose to earlier progression of leukemic disease, and result in a higher rate of CLL-related deaths.
\end{abstract}

Abbreviations: CLL: Chronic Lymphocytic Leukemia; ECOG: Eastern Cooperative Oncology Group; KPS: Karnofsky Performance Scale; IGHV; Immunoglobulin Heavy Chain Variable Region; CIRS: Cumulative Illness Rating Scale; GFR: Glomerular Filtration Rate; FCR: Fludarabine, Cyclophosphamide and Rituximab; FDA: US Food and Drug Administration; CRASH: The Chemotherapy Risk Assessment Scale for High-Age Patients; AE: Adverse Effects; Clb: Chlorambucil; CIT: Chemoimmunotherapy; PFS: Progression-Free-Survival

\section{Introduction}

CLL is one of the most common hematological malignancies in western countries [1]. It is the most common leukemia in adults in the Western world, accounting for approximately $11 \%$ of all hematological cancers [2]. Its prevalence has approached 120,000 , and over two thirds are over the age of 65 [3]. According to the most recent statistics, there are approximately 15,000 new cases of CLL in the United States every year, and half of them occur in individuals 71 years and older [4].

The median age at the time of diagnosis is 72 years, with $40 \%$ of patients are older than 75 years and $22.8 \%$ are 80 years old or more [2]. Persons 65 years of age and older account for $60 \%$ of the annual deaths attributed to CLL and for $70 \%$ of the approximately 150,000 CLL patients currently alive. With the rapid aging of the population, the incidence and prevalence of CLL among older persons is expected to continue to increase [4]. Although the disease remains incurable, important progress has been made [2]. Treatment advances that are prolonging the survival of the majority of patients will contribute to increased CLL survival in this population [4].

The disease is characterized by heterogeneous clinical course and outcome [1]. The natural history of CLL varies considerably from patient to patient [5]. During the last 15 years, several clinical, biological and molecular prognostic factors have been validated and some of them are currently used in patients' treatment management [1].

Factors known to influence a patient's clinical course and prognosis include clinical staging (Rai or Binet), serum markers (beta-2 microglobulin), IGHV mutational status, cytogenetic aberrations detected on FISH (e.g. del (17p), trisomy 12, $\operatorname{del}(13 q)$, del(11q)), and recurrent mutations in NOTCH1, SF3B1, and BIRC3. Cancer Network (NCCN) clinical practice guidelines do not recommend utilizing prognostic factors to determine treatment choices, with the exception of del (17p) and del (11q) [5].

\section{Age As A Prognostic Factor}

Age is associated with poor prognosis in CLL patients, with age and prognosis correlated at least up to 85 years of age. CLL is a cause of death for older individuals, especially for those aged 80 years and older [4]. Physiologic age, expressed as a frailty index, is associated with poor treatment tolerance. These findings raise the question of whether poorer survival in older patients is due to the biology of the disease or to poorer treatment tolerance. It was demonstrated in a previous study that age was a prognostic factor for poorer survival independent of the absence of IGHV mutation and of the p17 deletion. Age does not appear to be associated with decreased prevalence of either IGHV mutation 


\section{Cancer Therapy \& Oncology International Journal}

or increased prevalence of p17 deletion. No information is available concerning the association of age with CD38, ZAP-70, 11q deletion, or risk of Richter's transformation [4].

\section{Tools For Assessing Comorbidities}

All individuals 70 years of age and older should undergo a comprehensive geriatric assessment before initiation of therapy to estimate cancer-independent mortality risk and tolerance of chemotherapy and to identify conditions that may interfere with the treatment [6]. Several tools are available for assessment of comorbidities, functional status, and risk of chemotherapy toxicity for elderly patients with cancer [7].

Validated clinical tools, such as the NCI Comorbidity Index, the Charlson Comorbidity Index,and the CIRS, measure chronic illness burden and severity of chronic diseases [8]. CIRS and the Charlson Index correlate closely with each other, and both were reliable tools in trials of older cancer patients, e.g., the CLL8 and CLL11 trials. The CRASH score is helpful for evaluating risk of hematologic and non-hematologic toxicities from chemotherapy in patients 70 years and older [7].

American clinicians use end organ function, specifically GFR and liver function testing, and relevant cytogenetics, such as del (17p), when determining the type and intensity of upfront therapy. European practitioners more frequently use the CIRS to identify patients more appropriate for intensive CIT [3].

The German CLL Study Group used kidney function (< or >70 $\mathrm{ml} / \mathrm{min}$ ) and CIRS scores (< or $>6$ ) to stratify patients into 'go', 'slow-go' or 'no-go' categories. Elderly patients who were 'fit' were eligible for standard care treatment ('go'). In unfit patients, the effectiveness of the CLL treatment needed to be balanced with the increased risks of toxic effects, so non-chemotherapy approaches were preferred ('slow-go'). 'Frail' patients suffered from severe comorbidities, had short life expectancy and were given only conservative treatment ('no-go') [2].

\section{Treatment Goals In Elderly Cll Patients}

Treatment goals in these patients should aim for symptom control and improvement of quality of life rather than for induction of high CR rates. Aggressive therapy is typically not well tolerated. Strong consideration should be given to healthrelated quality of life issues when choosing a treatment regimen [7]. There was a need to tailor treatment goals for this population to the individual patient's comorbidities and functional capacity [7].

\section{Fit, Above 65-Year Patients Without Del (17p)}

In the USA, practitioners frequently use an age cut-off of 65 years to determine suitability for more intensive CIT unless they have a very good ECOG or KPS performance status [3].

\section{Bendamustine \pm rituximab(BR)}

In a phase III trial (CLL10), BR has shown improved safety profile and similar PFS to FCR in the fit, above 65-year patients without $\operatorname{del}(17 \mathrm{p})$ (treatment- related mortality: 3.9\% [FCR] vs $2.1 \%[\mathrm{BR}])$ [6].

\section{For unfit patients, inappropriate for FCR-type treatment}

In Europe, Clb has been used frequently in this setting. In the United States, $\mathrm{Clb}$ has been used much less and many physicians have administered CD20 monoclonal antibodies [3]. Combination CIT comprising either anti-CD20 monoclonal antibodies obinutuzumab or ofatumumab plus $\mathrm{Clb}$ was approved by the FDA and may be more suitable for older patients with comorbidities [7].

\section{Dose-Reduced FCR}

FCR is considered standard in young fit patients, without $\operatorname{del}(17 p)]$ or mutated TP53. Its application in elderly or unfit patients has been restricted by substantial myelo-suppression and infections, and other complications that may be particularly severe and potentially lethal in older individuals leading to detrimental reductions in dose-intensity [2].

\section{Obinutuzumab-Clb}

In elderly patients (median age 73 years) with multiple debilitating comorbidities, the combination of obinutuzumab with $\mathrm{Clb}$ was associated with a clinically significant prolongation of PFS, increased CR, and an increased rate of MRD negativity, compared with rituximab plus $\mathrm{Clb}$ combination. Moreover, obinutuzumab plus $\mathrm{Clb}$ resulted in a significant OS benefit compared with Clbmonotherapy, suggesting that the induction of deeper remissions (ie, MRD negativity) could translate into a survival advantage even in frail patients [6]. The higher efficacy of obinutuzumab- Clb in phase 3 CLL11 trial led to approval of obinutuzumab-Clb for the upfront treatment of unfit patients [5].

\section{Ofatumumab-Clb}

Ofatumumab was licensed in combination with bendamustine or Clb based on the randomized phase 3 COMPLEMENT- 1 trial. This trial showed significantly higher efficacy with moderately increased toxicity of ofatumumab-Clb compared with $\mathrm{Clb}$ [9].

\section{Clb and rituximab}

Study of $\mathrm{Clb}$ and rituximab in 100 de novo CLL patients over the age of 70 yielded an ORR and CR rate of 84 and $10 \%$, respectively, with a markedly lower incidence of cytopenias (neutropenia in $41 \%$ of patients) than purine-analog based CIT. Another study on $\mathrm{Clb}$ and rituximab showed similar response rates with, a longer median PFS with rituximab maintenance (38.2 versus 34.7 months) [3].

French Innotive Leukemia Organization studied 201 patients aged 80 years or older between 2003 and 2013. The median CIRS comorbidity score was 5 and the median creatinine clearance was $48 \mathrm{~mL} / \mathrm{min}$ (Cockcroft-Gault formula). Therapy consisted of $\mathrm{Clb}$ alone $(45.3 \%)$ or rituximab $+\mathrm{Clb}(22.7 \%)$, rituximab + bendamustine $(10.4 \%)$ or rituximab + cyclophosphamide + 
dexamethasone (5.5\%). ORR was $66.2 \%$ with $31.8 \%$ clinical complete remission. The median overall and PFS from treatment initiation was 53.7 and 18.33 months respectively. These results suggest that treatment is feasible in this age group even with immunochemotherapy [10].

\section{Lenalidomide (LEN)}

An open-label, randomized, multicenter, phase 3, parallelgroup study was conducted evaluating LEN as first-line therapy for elderly CLL patients ( $\geqslant 65$ years) (the ORIGIN trial (NCT00910910)). LEN did not prolong PFS and was associated with a lower response rate, a higher incidence of grade $\geqslant 3 \mathrm{AEs}$ and a higher number of deaths compared with Clb. Although LEN demonstrated clinical activity in a subset of patients in this trial, LEN monotherapy is not recommended as first-line therapy for elderly and/or frail CLL patients [11].

\section{delalisib}

Ongoing trials are evaluating the usefulness of phosphatidyl-3 kinase delta inhibitor, idelalisib, both alone and in various combinations as frontline CLL treatment in frail and elderly patients [6]. There is a recent report of using single agent idelalisib in the upfront setting in CLL patients older than 65 years. Only $14 \%$ of the 37 enrolled patients harbored a $17 p$ deletion. Of the 27 evaluable patients, the ORR was $81 \%$ with 9 (33 \%) PRs and 13 (48 \%) PRs with lymphocytosis [3]. None of the clinical trials onidelalisib has specifically focused on above 75-80 years patients population (median age of relapsed pivotal phase III trials was 67 and 71, respectively), no geriatric assessment has been used [2]. However, patients on idelalisib should be evaluated frequently for hepatotoxicity, colitis, intestinal perforation, pneumonitis, and rash.

CLL withdel(11q) should receive an alkylating agent-based regimen as frontline therapy whenever possible. For patients 70 years or older, first-line options include obinutuzumab plus $\mathrm{Clb}$, ofatumumab plus Clb, or bendamustine plus rituximab [7].

Patients with del(17p): The Bruton tyrosine kinase inhibitor,ibrutinib, is the only approvedtherapy for use in patients with del(17p) and is indicated in the NCCN guidelines as a category- 1 recommendation for patients age $\geq 70$ years and with significant comorbidities [7]. Clinical trials with ibrutinib havenot specifically focused on above 75-80 years population of patients [2]. Patients on ibrutinibshould be evaluated frequently for low-grade bleeding, diarrhea, atrial fibrillation, and rash [7].

\section{Response Assessment}

Early detection of absence of MRD in elderly individuals may prompt early CIT cessation, and reducing the risk of treatmentrelated toxicity. Frail patients may benefit primarily from a lowintensity approach in which the therapeutic endpoint is not CR or MRD, but rather duration of remission, tolerability, and quality of life. MRD negativity should be regarded as a treatment goal only in the setting of a clinical trial. There is a need for greater representation of elderly and frail patients in randomized CLL clinical trials to assess the therapeutic goals of CR and MRD negativity in this patient population, particularly in the era of kinase inhibitor therapy [6].

\section{In the Relapsed/Refractory Setting}

Data regarding treatment of these patients population are rather limitedandvery challenging. The novel targeted small molecules interfering with $\mathrm{B}$ cell receptor signaling ibrutinib and idelalisib showed impressive efficacy and radically changed the treatment paradigms for relapsed/refractory disease [12]. Their excellent tolerability and effectiveness, relatively mild toxicity of these agents make them very good candidates for elderly /comorbid patients. Other options for relapsed/ refractory disease include alemtuzumab, ofatumumab, high dose glucocorticoids + rituximab [12] or bendamustine and rituximab regimen [13]. LEN demonstrated activity in phase 2 trials in in front-line therapy and in relapsed and/or refractory CLL patients [11].

\section{Future Therapy}

Several ongoing clinical trials are specifically accruing patients older than 65 years with the goal of improving current outcomes. Two large cooperative group trials in the United States are currently evaluating the superiority of targeted agents such as ibrutinib in the frontline setting in terms of duration of remission, survival, quality of life, and tolerability in older patients with comorbidities (BR vsibrutinibvsibrutinib plus rituximab [NCT01886872]). A promising new therapeutic interventions including second-generation BTK or PI3K $\delta$ inhibitors, anti-apoptotic inhibitors, targeted tumor specific cellular therapies, and others are undergoing evaluation in clinical trials [6].

\section{Conclusion}

Older individuals may benefit from more effective, novel treatment of CLL. Targeted agents appear to address elderly patients' unmet needs of improved efficacy and tolerability.

\section{Reference}

1. Visentin A, Frezzalo F, Imbergamo S, Trimarco V, Martini V, et al. (2016) Evaluation of integrated CLL scoring system (ICSS) in 420 patients with chronic lymphocytic leukemia. Blood 128: 5563.

2. Ysebaert L, Feugier P, Michallet AS (2015) Management of elderly patients with chronic lymphocytic leukemia in the era of targeted therapies. Current opinoncol 27(5): 365-370.

3. Bachow SH, Lamanna N (2016) Evolving strategies for the treatment of chronic lymphocytic leukemia in the upfront setting. CurrHematolMalig Rep 11(1): 61-70.

4. Balducci L, Dolan D (2015) Chronic lymphocytic leukemia in the elderly: epidemiology and proposed patient-related approach. Cancer Control 22(4 Suppl): 3-6.

5. Marini BL, Samanas L, Perissinotti AJ (2016) Expanding the armamentarium for chronic lymphocytic leukemia: A review of novel agents in the management of chronic lymphocytic leukemia. J Oncol Pharm Practice. 


\section{Cancer Therapy \& Oncology International Journal}

6. Barrientos JC (2015) Management of chronic lymphocytic leukemia in the elderly. Cancer Control 22 (4Suppl): 17-23.

7. Pinilla-Ibarz J, Emole J (2015) Chronic lymphocytic leukemia in the elderly, which investigations are necessary: a map for the practicing oncologist. Cancer Control 22 (4 Suppl): 7-16.

8. Gupta NK, Andreadis C (2014) New Meets Old: A case study and review of novel therapeutics for the treatment of CLL in older patients. JNCCN 12 (10):1371-1375

9. Stilgenbauer S (2015) Prognostic markers and standard management of chronic lymphocytic leukemia. ASH Education Book 1: 368-377.

10. Meunier G, Ysebaert L, Nyuyen-Thi PL, Lepretre S, Quinquenel J, et al. (2016) First line therapy for chronic lymphocytic leukemia in patients older than 79 years is feasible and achieves good results: A FILO retrospective study. HematolOncol.

11. Chanan-Khan A, Egyed M, Robak T, Martinelli de Oliveira FA, Echeveste MA, et al. (2017) Randomized phase 3 study of lenalidomide versus chlorambucil as first-line therapy for older patients with chronic lymphocytic leukemia (the ORIGIN trial). Leukemia pp:1-3.

12. Smolei L (2016) Therapeutic approach to patients with chronic lymphocytic leukemia and significant comorbid conditions. Curr Cancer Drug 16(8): 710-720.

13. Smolei L (2015) Therapeutic options for "Slowgo" patients with chronic lymphocytic leukemia. KlinOnkol 28Suppl 3: 3S30-S38.

\section{Your next submission with Juniper Publishers} will reach you the below assets

- Quality Editorial service

- Swift Peer Review

- Reprints availability

- E-prints Service

- Manuscript Podcast for convenient understanding

- Global attainment for your research

- Manuscript accessibility in different formats

( Pdf, E-pub, Full Text, Audio)

- Unceasing customer service

Track the below URL for one-step submission https://juniperpublishers.com/online-submission.php 